\title{
Prolapsed Submucous Uterine Fibroid with Associated Uterovaginal Prolapse: A Case Report
}

\author{
Babah O A ${ }^{1}$, Afolabi B B ${ }^{2}$, Ayanbode $\mathrm{O}^{1}$, Atoki $\mathrm{A}^{1}$, Okafor $\mathrm{O}^{1}$. \\ ${ }^{1}$ Department of Obstetrics \& Gynaecology, Lagos University Teaching Hospital, PMB 12003, Idi-Araba, \\ Surulere, Lagos, Nigeria \\ ${ }^{2}$ Department of Obstetrics \& Gynaecology, Faculty of Clinical Sciences, College of Medicine, University of \\ Lagos/ Lagos University Teaching Hospital, Lagos, Nigeria
}

\begin{abstract}
:
Introduction: Prolapsed submucous uterine myoma occurring in association with uterovaginal prolapse is a rare gynaecological condition. When ulcerated, it may give an appearance that mimicks a malignant mass giving a sinister picture despite its benign nature. Only a few cases of prolapsed myoma has been reported, majority of which occurred in association with chronic non-puerperal uterine inversion. A few others were associated with urethrovaginal fistula and intermittent acute urinary retention.

Case: We present here, another rare case associated with uterovaginal prolapse. Mrs I.R. presented with a prolapsed submucous fibroid which appeared as a fungating ulcerated mass associated with a third degree uterovaginal prolapse. This finding together with the history of chronic weight loss she presented with, gave a false impression of a malignant tumour as a possible differential diagnosis. She had a polypectomy, followed by vaginal hysterectomy and bilateral salpingo-oophorectomy. Histopathology confirmed the mass to be a necrotic submuсоus leiomyoma.
\end{abstract}

Conclusion: It is thus important to have a prolapsed uterine fibroid as a differential whenever a mass protruding from the vagina is found in association with uterovaginal prolapse.

Key words: prolapsed myoma, uterovaginal prolapse.

\section{Introduction}

Leiomyomata are the commonest benign uterine tumour in the blacks. Sometimes submucous myomata may prolapse through the cervix into the vagina in which case they are referred to as prolapsed myoma. This is thought to be as a result of the gravitational pull of fundal submucous myoma when they grow large and heavy and is believed to be a gradual process. ${ }^{1}$

Women with prolapsed myoma may present with abnormal vaginal bleeding, dysmenorrhoea and large mass protruding from the cervix. ${ }^{2}$ Sometimes these prolapsed myomata may become necrotic and infected giving a sinister picture that may mimick a malignancy, thereby making diagnosis difficult.

A few cases of prolapsed uterine fibroids have been reported in the literature, although the incidence is unknown. Complications that may be associated with prolapsed myoma include uterine inversion due to the gravitational pull on the uterus, and urethrovaginal fistula and acute urinary retention as a result of the compression effect of the mass. In pregnancy, they may cause spontaneous miscarriage, premature rupture of fetal membranes and premature delivery.

Most cases of prolapsed submucous myoma reported in the literature were associated with chronic nonpuerperal uterine inversion. ${ }^{1.4} \mathrm{Lal} \mathrm{S}$ et al reported a case of huge degenerated prolapsed cervical myoma with associated urethrovaginal fistula. ${ }^{5}$ Eigbefoh $\mathrm{J}$ et al reported a case of prolapsed submucous fibroid polyp associated with intermittent acute urinary retention. ${ }^{6}$ Prolapsed myoma have also been reported in pregnancy. Two such cases, one cervical and the other submucousal, were recently reported by Kilpatrick CC et al. ${ }^{7} \mathrm{We}$ present here another rare case of a prolapsed submucous myoma with associated uterovaginal prolapse.

\section{Case Report}

Mrs I.R. is a 42 year old, Para 5+1 hairdresser, who presented at the Lagos University Teaching Hospital on the $3^{\text {rd }}$ of January, 2013 with a 3 year history of abnormal vaginal bleeding and protrusion of mass from the vagina and a 6 month history of progressive weight loss and foul smelling watery vaginal discharge. The abnormal vaginal bleeding was said to be in form of menorrhagia and intermenstrual bleeding. There was no associated abdominal distension, vomiting or change in bowel habit. There was no history of chronic cough.

She had earlier presented at a private hospital where she was transfused with 2 pints of blood because of haematocrit of $11.5 \%$. She also had urethral catheter passed at the same hospital because of urinary retention. She was not aware of and had never had Pap smear done. She has had 5 previous vaginal deliveries. 
Examination revealed a chronically ill-looking woman, afebrile (temperature $36.9^{\circ} \mathrm{C}$ ), anicteric, moderately pale, mildly dehydrated with no pedal oedema. Her pulse rate was 78 beats per minute and her blood pressure was 130/70mmHg. Only the first and second heart sounds were heard on auscultation. Findings on chest examination were essentially normal. There was no significant finding on abdominal examination. Pelvic examination revealed uterine procidentia with prolapsing fungating mass of about $12 \times 15 \mathrm{~cm}$ with a stalk arising from the endocervical canal. The stalk measured $4 \mathrm{~cm}$ in length and $3 \mathrm{~cm}$ in width. Copious foul smelling discharge was noted around the vulva, with excoriations extending to the peri-anal region.

The clinical impression was third degree uterovaginal prolapse with a fungating endocervical polyp likely fibroid, to rule out endometrial carcinoma. On investigation, her haematocrit was $15 \%$, total white blood cell count was 10,300 cells $/ \mathrm{mm}^{3}$ with neutrophils $73.7 \%$, lymphocytes $16.3 \%$, eosinophils $2.3 \%$ and basophils $0.4 \%$, platelet count was 357,000 cells $/ \mathrm{mm}^{3}$. Her serum electrolytes, urea and creatinine levels were essentially normal. Abdominopelvic ultrasonography revealed moderate calyceal dilatation bilaterally, with the mid-portion of the uterus folded on itself. Electrocardiography revealed non-specific $T$-wave inversion in $V_{1}-V_{4}$, otherwise normal findings. Chest radiograph was normal. She was negative for hepatitis B surface antigen, hepatitis $\mathrm{C}$ virus and Human Immunodeficiency Virus (HIV) I and II.

She had three (3) pints of blood transfused on admission. She also had intravenous ciprofloxacin and metronidazole, and oral diclofenac and ferrous gluconate. Wound dressing of the vulval excoriations was commenced with sofratulle gauze initially and later with zinc oxide cream. She was counseled for vaginal hysterectomy. Her post transfusion haematocrit was 32\%.

She had surgery a week after admission. Findings at surgery were a third degree uterovaginal prolapse with a prolapsed endocervical polyp approximately $10 \mathrm{x} 15 \mathrm{~cm}$ in size with a thick stalk about $4 \mathrm{~cm}$ thick and $4 \mathrm{~cm}$ long, oedematous cervix with normal size uterus. She had a polypectomy, followed by vaginal hysterectomy with and bilateral salpingo-oophorectomy. The estimated blood loss at surgery was approximately $700 \mathrm{mls}$. She had a pint of blood transfused because of post-operative haematocrit of $22 \%$. She was discharged home on the fifth post-operative day in satisfactory condition.

She was seen at the gynaecology out-patient clinic three (3) weeks later and her clinical condition was found to be satisfactory. Her histopathology revealed normal uterus, ovaries and Fallopian tubes, acute-onchronic cervicitis and necrotic submucous leiomyomata. Her subsequent follow up visits have been uneventful.

Figure 1 - Patient with prolapsed submucous myoma and associated uterovaginal prolapse. Soffratulle gauze over necrotic mass and zinc oxide paste applied on decubitus ulcer and adjacent areas. Urethral catheter in-situ.

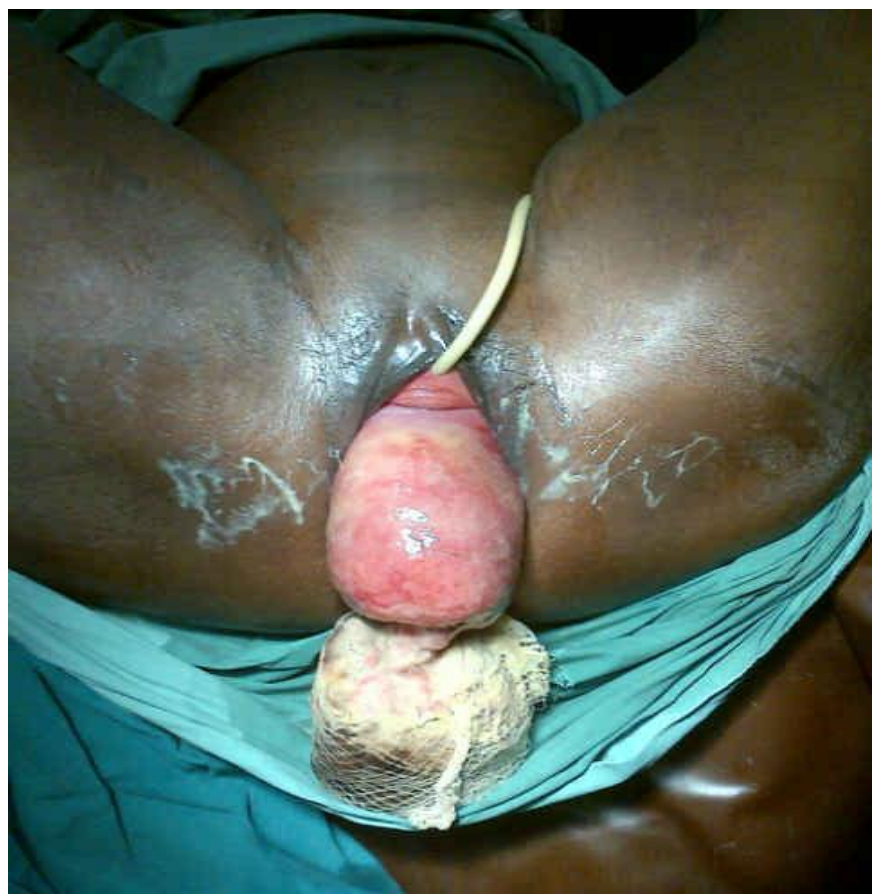




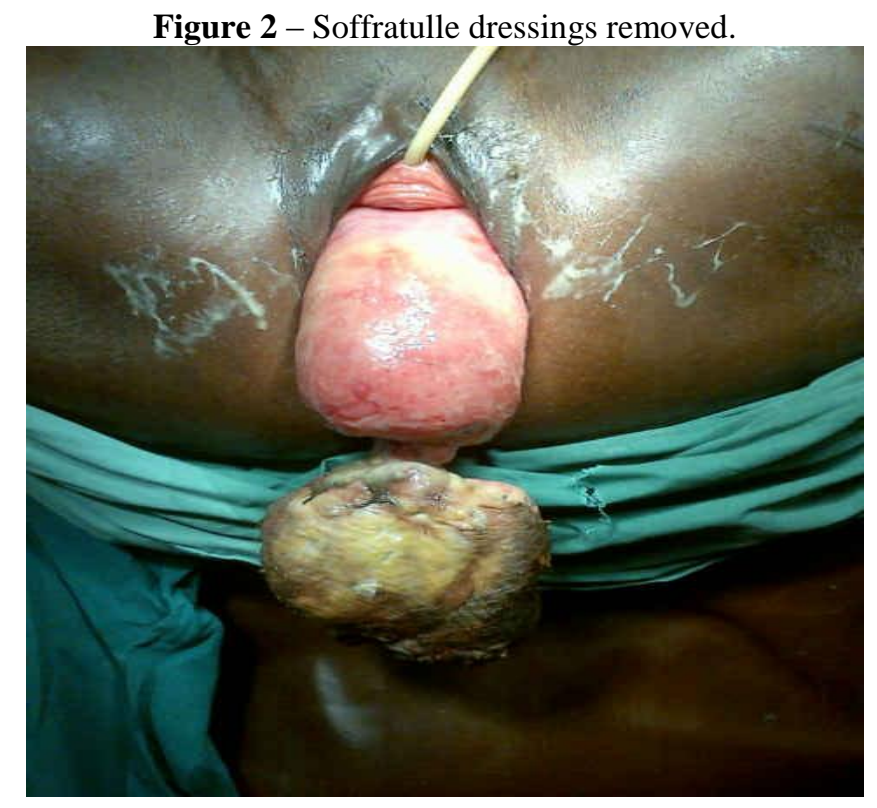

Figure 3 - Uterovaginal prolapse after vaginal polypectomy (just before performing a vaginal hysterectomy and bilateral salpingo-oophorectomy).

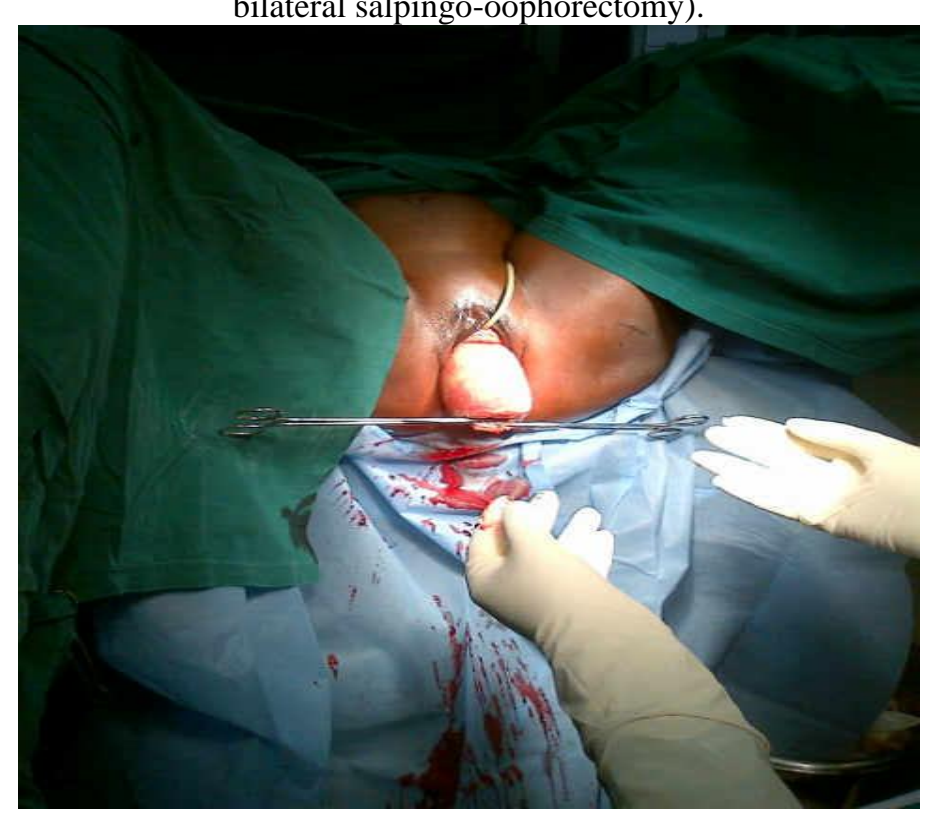

III. Discussion

Prolapsed submucous myoma is rare because often times a submucous myoma would have started manifesting symptoms such as menorrhagia even before it grows to a large size.

Prolapsed submucous myomata are thought to cause uterine inversion because of the gravitational pull on the uterine fundus. They are also thought to cause urethrovaginal fistula and acute urinary retention when they exert compression effect on surrounding structures. Our patient had acute urinary retention necessitating urethral catheterization at the referral centre. The ultrasonographic description of the uterus as "folding on itself at the mid portion" most likely suggests that our patient had chronic uterine inversion as well. Whether or not a prolapsed submucous myoma can be a single causative factor for uterovaginal prolapse is not clear.

The most distinctive feature in our patient is that she had a prolapsed submucous myoma in association with uterovaginal prolapse. We think this may be as a result of the persistent tension on the uterine supports (uterosacral and cardinal ligaments) caused by the graviatational pull of the large prolapsed submucous myoma. However we cannot say precisely if this is the major reason why this patient had the uterovaginal prolapse because she had other risk factors such as multiparity. It is possible though that the prolapsed submucous myoma further weakened an already compromised uterine support. 
Although we had a prolapsed submucous myoma as our diagnosis, the clinical picture of chronic weight loss. foul smelling watery vaginal discharge and severe anaemia also gave an impression of a possible malignant condition such as endometrial carcinoma or cervical cancer as a possible differential diagnosis. This is the reason why she was extensively evaluated pre-operatively to minimize risk of morbidities. The diagnosis was confirmed on histopathology.

Management options for prolapsed submucous myoma include vaginal myomectomy, abdominal myomectomy, abdominal hysterectomy usually following removal of the prolapsed myoma, and vaginal hysterectomy. ${ }^{8}$ The results of these treatment modalities has been good in cases reported so far. Our patient had a polypectomy followed by a vaginal hysterectomy and bilateral salpingo-oophorctomy and had a successful post-operative recovery.

It is thus important to have a prolapsed uterine fibroid as a differential whenever a mass protruding from the vagina is found in association with uterovaginal prolapse.

\section{Competing interests}

The authors declare that they do not have any competing interests.

\section{Authors' contributions}

Each of the authors of this manuscript were involved in the management of this case and also made significant contributions in the preparation of this manuscript and gave final approval of this version for publication.

\section{References}

[1]. Bista KD, Rana A, Guning G, Pradham N, Amatya A. Towards uterine inversion: Illustration of a gradual process through 3 cases of submucous myoma. N J Obstet Gynaecol. 2006; 1(2):51-54.

[2]. de Vries M, Perquin DAM. Non-puerperal uterine inversion due to submucous myoma in a young woman: a case report. Journals of Medical Case Reports. 2010; 4:21.

[3]. Omololu OM, Rabiu1 KA, Quadri MA, Oyedeko MO, Fatogun YM. Submucous fibroid: A case report. Nigerian Postgraduate Medical Journal. Npmj.edu.ng/sample-link/26-submucous-fibroid-a-case-report. Last updated 3/6/2011.

[4]. Kilpatrick CC, Chohan L, Maier RC. Chronic nonpuerperal uterine inversion and necrosis: a case report. Journal of Medical Case Reports. 2010; 4:381

[5]. Lal S, Sowmya SS, Kriplani A, Bhata N, Aganwal N. Urethrovaginal fistula due to prolapsed cervical myoma: A case report. The Internet Journal of Gynaecology and Obstetrics. 2007; 7(1): DOI:10.55 80/19 25.

[6]. Eigbefoh J, Okogbenin SA, Okogbo F, Eifediyi R, Omorogbe F, Isabu F. Prolapsed submucous uterine fibroid polyp associated with urinary incontinence: a case report. Sahel Medical Journal. ISSN:1118-856.

[7]. Kilpatrick CC, Adler MT, Chohan L. Vaginal myomectomy in pregnancy: a report of 2 cases. South Med J. 2010; 103(10):1058-1060.

[8]. Dicker D, Feldberg D, Dekel A, Yeshaya A, Samuel N, Goldman JA. The management of prolapsed submucous fibroids. Aust N Z J Obstet Gynaecol. 1986; 26(4):308-311. 